PACS: $07.20 . \mathrm{Dt} ; 52.25 . \mathrm{Rv} ; 44.10+\mathrm{i} ;$ 29.27.-a

\title{
TEMPERATURE MONITORING OF POLYMER FILMS UNDER IRRADIATION BY FAST ION BEAM
}

\author{
${ }^{1}$ V.N. Bondarenko, ${ }^{1}$ A.V. Goncharov, ${ }^{1}$ V.I. Sukhostavets, \\ ${ }^{2}$ T.Kh. Salikhov, ${ }^{2}$ A.A. Abdurahmonov \\ ${ }^{I}$ National Scientific Center "Kharkov Institute of Physics and Technology" \\ 1, Akademicheskaya st., Kharkov 61108, Ukraine \\ E-mail: goncharov@kipt.kharkov.ua \\ ${ }^{2}$ Tajik National University, Research Institute of Science \\ 17, Rudaki avenue, Dushanbe 734025, Tajikistan \\ E-mail:tsalikhov@mail.ru \\ Received December 27, 2016
}

A technique for infrared thermometry remote monitoring of temperature of area of a polymer film under ion irradiation is described. Calculation part of the technique takes into account the case when the irradiated area is significantly smaller than the instrument field of view. Temperature measurements were carried out with $20 \mu \mathrm{m}$ polyimide films irradiated by 1000 to $1600 \mathrm{keV}$ protons, with $1 \ldots 5 \mu \mathrm{A} / \mathrm{cm}^{2}$ current density. A model for calculation of temperature depth distribution in the irradiated film is developed. The model is also applicable for the case of ion irradiation in gaseous atmosphere, and takes into account temperature dependence of the polymer substance thermal conductivity, of the emissivity coefficient, and of the convective heat transfer coefficient. The calculated data are compared with the temperature measurements.

KEY WORDS: irradiation, fast ions, polyimide film, infrared thermometry

\section{КОНТРОЛЬ ТЕМПЕРАТУРИ ПОЛІМЕРНИХ ПЛВОК ПРИ ОПРОМІНЕННІ ПУЧКОМ ПРИСКОРЕНИХ ІОНІВ \\ ${ }^{1}$ В.М. Бондаренко, ${ }^{1}$ O.В. Гончаров, ${ }^{1}$ B.І. Сухоставець, ${ }^{2}$ T.X. Саліхов, ${ }^{2}$ А.А. Абдурахмонов \\ ${ }^{1}$ Національний Науковий Центр "Харківський фізико-технічний інститут”" вул. Академічна 1, м. Харків 61108, Украӥна \\ ${ }^{2}$ Таджикський національний університет, Науково-дослідний інститут пр. Рудакі, 17, м.Душанбе 734025,Таджикістан}

Розглянуто методику дистанційного контролю температури опромінюваної ділянки полімерної плівки за допомогою інфрачервоної термометрії. Розрахункова частина методики враховує також випадок, коли розміри опромінюваної ділянки значно менше поля зору термометра. На зразках поліімідних плівок товщиною 20 мкм проведені вимірювання температури на ділянці, що опромінювалась протонами в різних режимах (енергії протонів $1000 \ldots 1600$ кеВ, густина струму пучка $1 \ldots 5$ мкА $\left./ \mathrm{cm}^{2}\right)$. Розроблено модель для розрахунку розподілу температури по товщині опромінюваної плівки. Модель може застосовуватись і у випадку опромінювання у газовій атмосфері і враховує залежність від температури для коефіцієнта теплопровідності речовини полімеру, коефіцієнта чорноти поверхні та коефіцієнта тепловіддачі. Дані розрахунку порівнюються з даними вимірювань температури.

КЛЮЧОВІ СЛОВА: опромінення, прискорені іони, поліімідна плівка, інфрачервона термометрія

\author{
КОНТРОЛЬ ТЕМПЕРАТУРЫ ПОЛИМЕРНЫХ ПЛЕНОК ПРИ ОБЛУЧЕНИИ ПУЧКОМ УСКОРЕННЫХ ИОНОВ \\ ${ }^{1}$ В.Н. Бондаренко, ${ }^{1}$ А.В. Гончаров, ${ }^{1}$ В.И. Сухоставец, ${ }^{2}$ Т.Х. Салихов, ${ }^{2}$ А.А. Абдурахмонов \\ ${ }^{I}$ Начиональный научный иентр «Харьковский физико-технический институт» \\ ул. Академическая 1, г. Харьков 61108, Украина \\ ${ }^{2}$ Таджикский национальный университет, Научно-исследовательский институт \\ пр. Рудаки, 17, г. Душанбе 734025, Таджикистан
}

Рассмотрена методика дистанционного контроля температуры облучаемой области полимерной пленки с помощью инфракрасной термометрии. Расчетная часть методики учитывает и случай, когда размер облучаемой области значительно меньше поля зрения термометра. На образцах полиимидных пленок толщиной 20 мкм проведены измерения температуры в области пленки, облучаемой протонами в разных режимах (энергии протонов $1000 \ldots 1600$ кэВ, плотности тока пучка $1 \ldots 5$ мкА $/ \mathrm{cm}^{2}$ ). Разработана модель для расчета распределения температуры по толщине облучаемой пленки. Модель применима и для случая облучения в газовой атмосфере и учитывает зависимость от температуры для коэффициента теплопроводности вещества полимера, коэффициента черноты поверхности и коэффициента теплоотдачи. Данные расчета сопоставляются с данными измерений температуры.

КЛЮЧЕВЫЕ СЛОВА: облучение, ускоренные ионы, полиимидная пленка, инфракрасная термометрия

Пучки ускоренных ионов широко применяются для модификации свойств полимеров: изменения их электрических, адгезионных, прочностных и прочих характеристик [1-3]. В результате радиолиза происходит также изменение элементного состава полимерных материалов [2,4].

Известно, что от температуры полимера во время облучения существенно зависит подвижность свободных радикалов и газообразных продуктов, образующихся под действием облучения. С повышением плотности тока пучка, а значит, и температуры образца при облучении, увеличивается электропроводность облученного полимера [1]. Кроме того, с повышением температуры полимера может происходить изменение его структуры. 
В частности, для полиимида такой характерной температурой является температура стеклования (321 $\left.{ }^{0} \mathrm{C}[5]\right)$.

В конечном счете, эти температурные эффекты могут существенно сказываться на характеристиках облученных материалов [1, 3, 6-8]. В работе [9] отмечено, что с точки зрения исследования механизмов собственно радиационных эффектов в полимере проведение измерений при высоких температурах не очень желательно, так как в этом случае относительный вклад радиационных эффектов в эволюцию структуры облучаемого материала снижается.

Таким образом, с точки зрения воспроизводимости экспериментальных результатов в опытах по облучению полимеров важен контроль не только флюенса облучения, но и температуры образца в зоне облучения в самом процессе опыта.

В случаях, когда размеры образца достаточно велики, наиболее удобным является дистанционный контроль его температуры по ИК излучению с поверхности данного объекта. Так, например, в работе [10] эта методика применялась для контроля температуры свариваемых стыков конструкций сверхпроводящих резонаторов в процессе остывания после электронно-лучевой сварки.

А в работе [11] таким методом контролировалась температура образцов, облучаемых сканируемым пучком электронов с энергией 10 МэВ и мощностью 10 кВт. При этом размеры облучаемой области были достаточно большими - 20 см и более.

Однако применение такого рода контроля существенно усложняется, если размеры области, в которой происходит выделение энергии, заметно меньше поля зрения ИК термометра.

В настоящей работе были поставлены следующие цели: а) разработка методики дистанционного контроля температуры именно для такого случая, а конкретно для контроля температуры облучаемой ионным пучком области полимерной пленки, когда размеры этой области не превышают нескольких мм; б) разработка теоретической модели, описывающей распределение температуры в облучаемой области пленки. Первая часть работы была выполнена в ННЦ ХФТИ, а вторая - в Таджикском национальном университете.

\section{ЭКСПЕРИМЕНТ И МЕТОДИКА ОПРЕДЕЛЕНИЯ ТЕМПЕРАТУРЫ}

Исследование температурных режимов облучаемых полимерных пленок проводились на универсальной камере для ядерно-физических методов анализа [12] на протонном пучке ускорителя “Сокол” ННЦ ХФТИ (рис. 1).

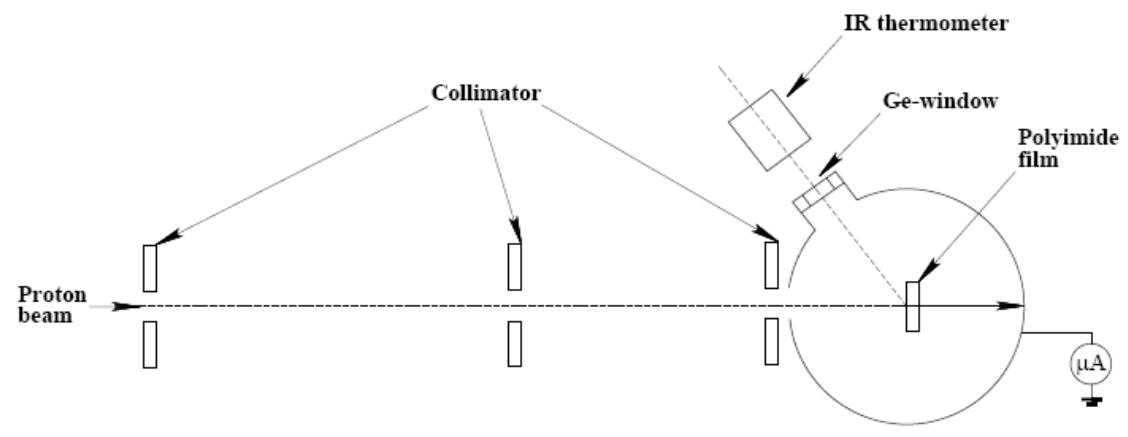

Рис. 1. Схема измерений температуры поверхности облучаемой полимерной пленки.

Пучок ускоренных протонов сквозь систему круглых диафрагм проходил в камеру мишени, электрически соединенную с держателем мишени. Поскольку размеры камеры были существенно больше размеров облучаемой области мишени, то вторичные электроны, образующиеся при бомбардировке поверхности мишени, стекали на внутреннюю поверхность камеры. В стенке камеры тормозились и протоны, прошедшие сквозь пленку. Камера была соединена с землей через интегратор тока. Такая геометрия позволяла контролировать средний ток пучка $I=Q / t$, где $Q$ - регистрируемый интегратором стекающий заряд, $t-$ время экспозиции.

Откачка камеры до остаточного давления $5 \cdot 10^{-4}$ Па обеспечивалась турбомолекулярным насосом и азотной ловушкой, расположенной над держателем мишени.

В настоящей работе в качестве мишеней-образцов использовались полиимидные пленки марки ПМ толщиной $L=20$ мкм. Полиимиды относятся к термостойким полимерам. Внутренний диаметр держателя, в котором закреплялась пленка, составлял 19 мм. Центр держателя и, соответственно, центр мишени совпадали с центром камеры.

Облучение проводилось протонами с энергией 1,0..1,6 МэВ. Плоскость мишени была перпендикулярна направлению пучка. Камера юстировалась по отношению к пучку таким образом, чтобы пучок попадал на центр мишени. 
Измерения размеров облученной области, проводившиеся с помощью фотометрического метода, показали, что средний диаметр облучаемого пятна изменялся в пределах 1,8...2,2 мм в зависимости от режима работы ускорителя. С учетом вариации тока пучка в пределах $30 \ldots 150$ нА это соответствовало плотностям тока пучка на мишени в пределах $1 \ldots 5$ мкА/см².

Измерение температуры в облучаемой области полиимидного образца осуществлялось дистанционно с помощью инфракрасного термометра “Fluke576CF”, регистрирующего тепловое излучение от мишени. Согласно паспортным данным, спектральный диапазон длин волн инфракрасного излучения, в котором работает прибор, составляет 8..14 мкм, а погрешность определения температуры - 0,75 \% [13].

Поскольку образец находился в вакууме, то излучение от мишени к прибору выводилось сквозь $G e$-окно. Пропускная способность германиевых окон в указанном рабочем диапазоне длин волн очень слабо зависит от длины волны.

ИК-термометр был расположен под углом $\varphi=40^{0}$ относительно нормали к мишени и установлен на расстоянии 300 мм от мишени. Согласно техническому описанию на таком расстоянии диаметр поля зрения прибора составляет 6 мм, что заметно больше размера облучаемого пятна на мишени. С помощью специальной трехлучевой лазерной подсветки прибора его можно было отъюстировать по отношению к облучаемому пятну таким образом, чтобы оно находилось в центре поля зрения прибора. Эта операция проводилась на атмосфере в отсутствие $G e$-окна.

Таким образом, в данной геометрии прибор регистрировал ИК-излучение как от облучаемой области, так и от окружающей ее не облучаемой области пленки, что является основной трудностью методики измерения, использованной в настоящей работе. Поэтому возникает необходимость в построении модели, с помощью которой можно было бы определить искомую среднюю температуру $T_{\text {irrad }}$ в облучаемой области мишени из данных измерения с помощью ИК-термометра.

Пусть $A_{\text {irrad }}$ - площадь облучаемой области мишени; $T_{\text {unirrad }}-$ температура в необлучаемой области; $A_{\text {unirrad }}-$ площадь этой области в пределах поля зрения ИК-термометра; $T_{\text {eff }}$ - некоторая эффективная температура, определяемая на основании мощности излучения, регистрируемой прибором со всего поля зрения; $A_{\text {total }}=A_{\text {irrad }}+A_{\text {unirrad. }}$ Будем считать, что в установившемся тепловом режиме отвод мощности из облучаемой области пленки происходит главным образом за счет теплового излучения. В нашем случае это оправдано тем, что теплопроводность полимеров низка, а расстояние от данной области до металлического держателя много больше ее размеров. В соответствии с законом Стефана-Больцмана, поток теплового излучения от поверхности твердого тела пропорционален $T^{4}$, где $T$ - абсолютная температура тела. Тогда, применительно к нашему случаю, это приводит к соотношению

$$
A_{\text {total }} T_{\text {eff }}^{4}=A_{\text {irrad }} T_{\text {irrad }}^{4}+A_{\text {unirrad }} T_{\text {unirrad }}^{4} .
$$

В обеих частях этого соотношения опущены такие постоянные, как $\sigma$ - постоянная Стефана-Больцмана, $\alpha-$ коэффициент черноты излучающей поверхности (для полимеров эта величина близка к единице), постоянная пропускания $G e$-окна и геометрический фактор $\cos \varphi$, обусловленный тем, что ИК-термометр расположен под углом относительно нормали к излучающей поверхности.

Очевидно, что если температура в облучаемой области мишени заметно превышает температуру в области не облучаемой, то второй член в правой части соотношения (1) много меньше первого члена.

Анализ показывает, что основной погрешностью в выражении (1) является неопределенность в измерении радиуса облучаемой области. Она составляла порядка $10 \%$, что приводило к погрешности искомой температуры $T_{\text {irrad }}$ до 20-30 градусов.

В таблице 1 представлены характеристики режимов облучения.

Таблица 1.

Проективные пробеги протонов в полиимиде, рассчитанные с помощью программы SRIM [14], сбросы их энергии в 20-микронной полиимидной пленке и использовавшиеся режимы облучения.

\begin{tabular}{|c|c|c|c|c|}
\hline $\begin{array}{c}\text { Энергия } \\
\text { протонов, } \\
\text { кэВ }\end{array}$ & $\begin{array}{c}\text { Проективный пробег } \\
\text { протонов, мкм }\end{array}$ & $\begin{array}{c}\text { Сброс энергии протонов } \\
\text { в пленке, кэВ }\end{array}$ & $\begin{array}{c}\text { Эокерговыделение } \\
\text { пучка в пленке, } \\
\text { мВт/мм }^{2}\end{array}$ \\
\hline 1000 & 19,2 & 1000 & $30,60,90$ & $10,19,29$ \\
\hline 1200 & 25,6 & 774 & $30,60,90,120$ & $7,15,22,30$ \\
\hline 1400 & 32,6 & 638 & $30,60,90,120,150$ & $6,12,18,24,31$ \\
\hline 1600 & 40,4 & 558 & $30,60,90,120,150$ & $5,11,16,21,27$ \\
\hline
\end{tabular}

Энерговыделение пучка рассчитывалось, исходя из плотности тока пучка и сброса энергии протонов в пленке.

Таким образом, лишь при энергии 1000 кэВ протоны не проходили сквозь использовавшуюся полиимидную пленку.

Температура $T_{\text {irrad }}$ может быть также оценена из баланса между поглощаемой и излучаемой мощностью. Если предположить, что температуры лицевой и тыльной поверхностей пленки в области облучения 
одинаковы, то радиационное охлаждение образца будет происходить с обеих этих поверхностей с одинаковой интенсивностью. Тогда в установившемся режиме средняя температура в облучаемой области может быть оценена из равенства мощности пучка, поглощаемой в пленке, и мощности, излучаемой по закону СтефанаБольцмана:

$$
I \Delta E / e=2 \alpha \sigma\left(T_{\text {irrad }}^{4}-T_{0}^{4}\right) A_{\text {irrad }},
$$

где $I$ - ток пучка; $\Delta E$ - сброс энергии ионов в материале пленки; $e$ - заряд электрона; $T_{0}-$ исходная температура образца (комнатная температура в К).

Очевидно, что в соотношении (2) не учтен сток тепла от облучаемого объема к держателю образца за счет

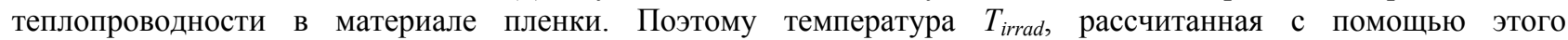
соотношения, является заведомо завышенной, но, в принципе, может приближаться к искомой в случае большого энерговыделения в облучаемой области (т.е. в случае больших плотностей тока пучка).

\section{ВЛИЯНИЕ ТЕМПЕРАТУРНОЙ ЗАВИСИМОСТИ ТЕПЛОФИЗИЧЕСКИХ ВЕЛИЧИН НА СТАЦИОНАРНОЕ ТЕМПЕРАТУРНОЕ ПОЛЕ В ТОНКИХ ПОЛИМЕРНЫХ ПЛЕНКАХ ПОД ИОННЫМ ОБЛУЧЕНИЕМ}

В данном разделе описана модель для расчета распределения температуры по толщине облучаемой тонкой полимерной пленки. Ниже под тонкими пленками будем подразумевать такие, толщины $L$ которых меньше длины $R$ проективного пробега ионов в веществе пленки.

Будем рассматривать установившийся, стационарный режим облучения, когда время облучения много больше времени тепловой релаксации.

Для общности будем считать, что облучаемый образец пленки находится в газообразной среде (такой случай может быть реализован, например, при выводе ионного пучка в атмосферу).

Также примем во внимание зависимость от температуры $T$ для коэффициента теплоотдачи $h=h(T)$, коэффициента теплопроводности $\kappa=\kappa(T)$ вещества образца и коэффициента черноты поверхности образца полимера $\alpha=\alpha(T)$.

Пусть однородный по поперечному сечению поток быстрых ионов с энергией $E$ падает на мишень перпендикулярно ее поверхности вдоль оси $X$. Будем считать, что сток тепла от облучаемой области образца на держатель пленки в направлении, перпендикулярном оси $X$, пренебрежимо мал по сравнению с исходящим от облучаемой области потоком теплового излучения. Тогда задача сводится к одномерной, и в рассматриваемом стационарном случае справедливо следующее нелинейное уравнение теплопроводности [15]:

$$
\frac{d}{d x}\left[\kappa(T) \frac{d T}{d x}\right]=-\frac{G(L, R) E J}{e R},
$$

где $T(x)$ - распределение абсолютной температуры по толщине пленки; $J$ - плотность потока ионов; $e-$ заряд электрона. Величина $G(L, R)$ описывает фактор уменьшения величины энергии, переданной ионом пленке, в зависимости от $R=R(E)$. В грубом приближении эту величину можно представить в виде $G(L, R) \approx L / R$.

Два граничных условия, учитывающих тепловое излучение и конвективное охлаждение с обеих поверхностей пленки, можно записать в виде [16]:

$$
\begin{gathered}
{\left.\left[\kappa(T) \frac{d T}{d x}\right]\right|_{x=0}=\left.\left[h(T)\left(T-T_{0}\right)+\sigma\left(\alpha(T) T^{4}-\alpha\left(T_{0}\right) T_{0}^{4}\right)\right]\right|_{x=0},} \\
{\left.\left[-\kappa(T) \frac{d T}{d x}\right]\right|_{x=L}=\left.\left[h(T)\left(T-T_{0}\right)+\sigma\left(\alpha(T) T^{4}-\alpha\left(T_{0}\right) T_{0}^{4}\right)\right]\right|_{x=L},}
\end{gathered}
$$

где $T_{0}$ - исходная температура.

Температурные зависимости для коэффициентов теплопроводности, черноты поверхности и теплоотдачи примем в следующем, линейном приближении:

$$
\begin{array}{cll}
\kappa(T)=\kappa_{0}\left[1+\delta_{2}\left(T-T_{0}\right)\right], & \kappa_{0}=\kappa\left(T_{0}\right), & \delta_{1}=\left.\frac{1}{\kappa_{0}} \frac{\partial \kappa}{\partial T}\right|_{T=T_{0}}, \\
\alpha(T)=\alpha_{0}\left[1+\delta_{3}\left(T-T_{0}\right)\right], & \alpha_{0}=\alpha\left(T_{0}\right), & \delta_{2}=\left.\frac{1}{\alpha_{0}} \frac{\partial \alpha}{\partial T}\right|_{T=T_{0}}, \\
h(T)=h_{0}\left[1+\delta_{4}\left(T-T_{0}\right)\right], & h_{0}=h\left(T_{0}\right), & \delta_{3}=\left.\frac{1}{h_{0}} \frac{\partial \kappa}{\partial T}\right|_{T=T_{0}} .
\end{array}
$$

Введем $\tau(x)=T(x)-T_{0}$. Соответственно, уравнение теплопроводности принимает вид: 


$$
\frac{d}{d x}\left[\left(1+\delta_{1} \tau\right) \frac{d \tau}{d x}\right]=-\frac{G(L, R) E J}{e \kappa_{0} R}=-2 F .
$$

Используя обозначение $z(x)=1+\delta_{1} \tau(x)$ найдем первый и второй интегралы уравнения (9):

$$
\frac{z}{\delta_{1}} \frac{d z}{d x}=C_{1}-2 F x, \quad z(x)=\left(2 \delta_{1}\left(C_{1}-F x\right) x+C_{2}\right)^{1 / 2},
$$

где $C_{1}$ и $C_{2}$ - постоянные интегрирования. Их можно выразить через установившиеся температуры $T_{1}$ и $T_{2}$, соответствующие лицевой и тыльной поверхностям пленки в облучаемой области, решая систему уравнений

$$
z(0)=1+\delta_{1} \tau_{1}=C_{2}^{1 / 2}, \quad z(L)=1+\delta_{1} \tau_{2}=\left(2 \delta_{1}\left(C_{1}-F L\right) L+C_{2}\right)^{1 / 2},
$$

где $\tau_{1}=T_{1}-T_{0}, \tau_{2}=T_{2}-T_{0}$. Температура $T_{1}$ эквивалентна температуре $T_{i r r a d}$, которой речь шла выше.

В результате, выражение, описывающее распределение температуры по толщине пленки в облучаемой области, принимает вид:

$$
\delta_{1} \tau(x)=-1+\left\{1+2 \delta_{1}\left[\tau_{1}\left(1+\frac{\delta_{1} \tau_{1}}{2}\right)\left(1-\frac{x}{L}\right)+\tau_{2}\left(1+\frac{\delta_{1} \tau_{2}}{2}\right) \frac{x}{L}+F(L-x) x\right]\right\}^{1 / 2} .
$$

При этом граничные условия (4-5) в новых обозначениях преобразуются в систему уравнений

$$
\begin{aligned}
& \tau_{1}-\tau_{2}+\frac{\delta_{1}}{2}\left(\tau_{1}^{2}-\tau_{2}^{2}\right)-F L^{2}+\frac{h_{0} L}{\kappa_{0}}\left(1+\delta_{3} \tau_{1}\right) \tau_{1}+\frac{\alpha_{0} \sigma L}{\kappa_{0}}\left[\left(1+\delta_{2} \tau_{1}\right)\left(\tau_{1}+T_{0}\right)^{4}-T_{0}^{4}\right]=0, \\
& \tau_{1}-\tau_{2}+\frac{\delta_{1}}{2}\left(\tau_{1}^{2}-\tau_{2}^{2}\right)+F L^{2}-\frac{h_{0} L}{\kappa_{0}}\left(1+\delta_{3} \tau_{2}\right) \tau_{2}-\frac{\alpha_{0} \sigma L}{\kappa_{0}}\left[\left(1+\delta_{2} \tau_{2}\right)\left(\tau_{2}+T_{0}\right)^{4}-T_{0}^{4}\right]=0 .
\end{aligned}
$$

С помощью численного решения данной системы нелинейных алгебраических уравнений для $\tau_{1}$ и $\tau_{2}$ можно найти зависимости этих величин от параметров падающего потока ионов.

Анализ системы уравнений (13-14) показывает, что величины $\tau_{1}$ и $\tau_{2}$ практически совпадают. При $\delta_{1} \tau_{1}<<1$ распределение (12) принимает форму, близкую к параболической:

$$
\tau(x) \cong \tau_{1}+F(L-x) x .
$$

Приближение (15) справедливо при условии $F L^{2}<<\tau_{1}$. Из (15) следует, что температура достигает максимума $\tau=\tau_{\max }$ при $x=L / 2$, т.е. в центре облучаемого объема пленки. С помощью этого приближения можно оценить разность между максимальной температурой и температурой на облучаемой поверхности:

$$
\tau_{\text {max }}-\tau_{1} \cong F L^{2} / 4
$$

Это значит, что данное различие в температурах монотонно растет с увеличением мощности, вводимой в облучаемую область, т.е., в конечном счете, с увеличением температуры на облучаемой поверхности.

Приведем также приближенное выражение для искомой температуры $T_{1}$ для частного случая, когда облучение производится в вакууме. Тогда из уравнения (13) при условии $\tau_{2}=\tau_{1}$ следует:

$$
\left(1-\delta_{2} T_{0}+\delta_{2} T_{1}\right) T_{1}^{4} \cong\left(\frac{\kappa_{0} F L}{\alpha_{0} \sigma}+T_{0}^{4}\right)=H^{4} .
$$

При условии $\delta_{2} \tau_{1}<<1$ из этого уравнения вытекает приближенное решение:

$$
T_{1} \cong H\left(1-\delta_{2}\left(H-T_{0}\right) / 4\right) \text {. }
$$

Второй член в (18) представляет собой отрицательную поправку к первому приближению $T_{1} \approx H$. Поскольку произведение $\kappa_{0} F$ не зависит от $\kappa_{0}$, то температура $T_{1}$, определяемая выражением (18), также не зависит от характеристик теплопроводности. Выражение (18) через параметр $\delta_{2}$ температурной зависимости коэффициента черноты дает несколько меньшее значение температуры поверхности облучаемой области по сравнению с первым приближением и значением, которое следует из уравнения (2).

\section{РЕЗУЛЬТАТЫ И ИХ ОБСУЖДЕНИЕ}

При пересчете экспериментально измеренной температуры $T_{\text {eff }}$ в искомую температуру $T_{\text {irrad }}$ с помощью соотношения (1) предполагалось, что средняя температура $T_{\text {unirrad }}$ в не облучаемой области пленки близка к комнатной температуре $T_{0}$. Эти данные представлены в третьей колонке таблицы 2.

Расчет температуры поверхности пленки в облучаемой области с помощью соотношения (2) проводился при фиксированном значении 0,95 для коэффициента черноты поверхности полиимидной пленки. Результаты расчета даны в четвертой колонке.

В пятой колонке представлены результаты расчета температуры поверхности в облучаемой области с помощью рассмотренной выше теоретической модели, учитывающей температурные зависимости для коэффициента теплопроводности вещества образца и коэффициента черноты поверхности образца полимера. 
При расчете на основе решения системы уравнений (13-14) использовались следующие значения параметров: $L=20 \cdot 10^{-6} \mathrm{~m} ; h_{0}=0$ (поскольку облучение проводилось в вакууме); $\kappa_{0}=0,155 \mathrm{BT}^{\cdot} \mathrm{M}^{-1} \mathrm{~K}^{-1}$ и $\delta_{1}=8,4 \cdot 10^{-4} \mathrm{~K}^{-1}[17] ; \alpha_{0}=0,95$ и $\delta_{2}=1,4 \cdot 10^{-4} \mathrm{~K}^{-1}$ [10]. Параметры $E$ и $R$ заимствовались из таблицы 1 . При расчете плотности тока пучка предполагалось, что радиус облучаемой области равен 1 мм, т.е. среднему значению для радиусов характерных пятен, возникавших на пленке в процессе облучения в рассмотренных режимах.

Для энергии протонов 1000 кэВ расчет с помощью модели не является вполне корректным, т.к. в этом случае не соблюдалось условие $L<R$.

Таблица 2.

Результаты обработки измерений и результаты расчета.

\begin{tabular}{|c|c|c|c|c|}
\hline \multirow{2}{*}{$\begin{array}{c}\text { Энергия } \\
\text { протонов, } \\
\text { кэВ }\end{array}$} & \multirow{2}{*}{$\begin{array}{c}\text { Энерговыделение } \\
\text { пучка в пленке, } \\
\text { мВт/мм² }\end{array}$} & \multicolumn{3}{|c|}{ Температура облучаемой поверхности пленки, ${ }^{0} \mathrm{C}$} \\
\hline & & $\begin{array}{c}\text { определенная } \\
\text { с помощью соотношения } \\
\text { (1) }\end{array}$ & $\begin{array}{c}\text { вычисленная } \\
\text { с помощью соотношения } \\
\text { (2) }\end{array}$ & $\begin{array}{c}\text { вычисленная } \\
\text { из системы } \\
\text { уравнений (13-14) }\end{array}$ \\
\hline 1000 & $10,19,29$ & $195,292,340$ & $283,382,450$ & $291,374,464$ \\
\hline 1200 & $7,15,22,30$ & $204,276,354,374$ & $252,343,406,455$ & $248,322,387,434$ \\
\hline 1400 & $6,12,18,24,31$ & $191,278,317,343,377$ & $230,316,376,422,461$ & $212,280,338,382,420$ \\
\hline 1600 & $5,11,16,21,27$ & $204,239,292,327,364$ & $215,298,355,400,437$ & $184,245,299,340,374$ \\
\hline
\end{tabular}

Расчет показывает, что для всех использовавшихся режимов облучения имеет место $F L^{2}<<\tau_{1}$. Поэтому вычисляемая с помощью соотношения (16) разность между температурой в центре облучаемого объема пленки и температурой на облучаемой поверхности является пренебрежимо малой.

Естественно было предположить, что, в условиях конвертации потери энергии ионов в пленке преимущественно в тепловую энергию, точки, относящиеся к разным режимам облучения, должны в координатах температура/энерговыделение ложиться на некоторую универсальную кривую. Данные из таблицы 2 в графическом виде представлены на рис. 2, из которого видно, что экспериментальные точки действительно с некоторым разбросом группируются вокруг такой кривой. Систематически ниже ее расположены точки, соответствующие облучению при энергии протонов 1000 кэВ, при которой имеет место их полное замедление в материале пленки.

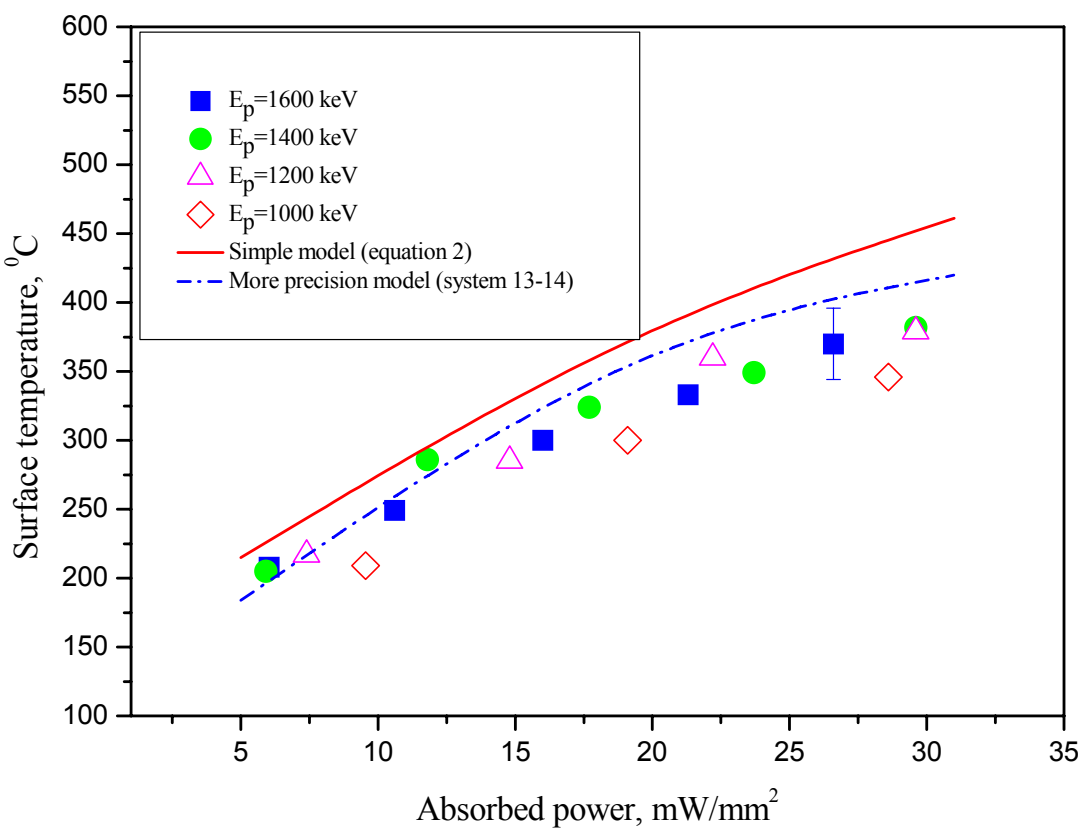

Рис. 2. Сравнение экспериментальных и расчетных зависимостей температуры поверхности облучаемой области пленки полиимида от энерговыделения протонного пучка для разных режимов облучения (см. табл.2).

Из данной универсальной зависимости следует, что если облучение полиимидной пленки необходимо проводить в области температур ниже температуры стеклования полиимида $\left(321^{0} \mathrm{C}[5]\right)$, то энерговыделение не должно превышать 17 мВт/мм².

Конечно, рассматриваемая модель не учитывает сток тепла к держателю через не облучаемую часть пленки за счет механизма теплопроводности. Но оценки показывают, что для размеров пленок, 
использовавшихся в настоящих измерениях, этот фактор не является значительным в силу низкого коэффициента теплопроводности полиимида.

Также оценки показывают, что унос энергии из полиимидной пленки за счет мгновенного излучения, например, эмиссии вторичных электронов с обеих поверхностей облучаемой области пленки или за счет выхода из облучаемого объема электромагнитного излучения в видимом, ультрафиолетовом и рентгеновском диапазоне, также является незначительным, хотя все эти типы эмиссии и растут пропорционально току пучка. Например, абсолютный световой выход даже специально допированного полиимида не превышает 0,3\% [18]. Поэтому в наших условиях погрешность теплового энерговыделения за счет этого фактора также не превышает этой величины, а соответствующая погрешность определения температуры не превышает 0,5 градуса.

Из рис. 2 видно, что согласие экспериментальной зависимости и теоретической зависимости, учитывающей температурные зависимости теплофизических характеристик облучаемого полимера, является вполне удовлетворительным. Несколько худшее согласие наблюдается с кривой, построенной на основании соотношения (2) с фиксированным значением $\alpha=0,95$. И этому есть простое объяснение: учет монотонного нарастания зависимости $\alpha(T)$ с температурой эквивалентен дополнительному увеличению эффективного значения $\alpha$, а рост $\alpha$ приводит к большей интенсивности радиационного охлаждения пленки.

Конечно, некоторое расхождение между данными измерений и данными расчета может быть связано и с различием между табличными данными по коэффициенту черноты поверхности и реальным значением этого параметра для использовавшегося образца полиимидной пленки.

\section{ВЫВОДЫ}

Рассмотренная методика контроля температуры в облучаемой области полимерной пленки, использующая инфракрасную термометрию, позволяет осуществлять это бесконтактным дистанционным образом в непрерывном режиме даже в случае, когда размеры облучаемой области заметно меньше поля зрения ИК термометра.

Предложенная модель расчета распределения температуры по толщине пленки, облучаемой потоком ионов, учитывает температурные зависимости теплофизических характеристик облучаемого полимера, что позволяет вычислить температуру поверхности облучаемой области пленки более корректным образом и объяснить некоторое различие в температурах между поверхностями облучаемой области пленки и ее заглубленными слоями. Это различие нарастает с увеличением мощности, вводимой в пленку при облучении.

Очевидно, что хотя измерения и конкретные расчеты были проделаны для протонного пучка, рассмотренные в данной работе экспериментальная и расчетная методики контроля температуры облучаемой пленки могут быть применены и для других типов ионов. Конечно же, условием применения расчетной методики остается критерий $L<R$.

Понятно, что описанные методики могут быть применены не только для случая облучения полимерных пленок, но и для тонких образцов любого материала с низкой теплопроводностью, например, для неметаллических материалов.

При сравнении экспериментальных и расчетных данных наблюдается небольшое систематическое расхождение. Причина этого не вполне ясна, поэтому необходимо дальнейшее продвижение как в совершенствовании процесса измерения, так и в развитии предложенной теоретической модели.

Авторы выражают признательность Зацу А.В., Кузьменко В.В. и Карпусю С.Г. за содействие в проведении измерений.

\section{REFERENCES}

1. Popok V.N., Azarko I.I., Haybullin R.I. Vliyanie vysokih doz implantacii i plotnosti ionnogo toka na svoystva plenok poliimida [Influence of high implantation dozes and ion current density on polyimide film properties] // ZnTF. - 2002. - Vol.72. - Iss.4.P.88-93. (in Russian)

2. Vencatesan T., Forrest S.R., Kaplan M.L., Murray C.A., Shmidt P.H., Wilkens B.J. Ion-beam-induced conductivity in polymer films // J. Appl. Phys. - 1983. - Vol.54. - No.6. - P.3150-3153.

3. Guseva M.I. Ionnaya implantaciya v metallah [Ion implantation in metals] // Poverhnost. Ser. Fizika, himiya, mehanika. - 1982. - No.4. - P.27-50. (in Russian)

4. Bondarenko V.N., Goncharov A.V., Sukhostavets V.I., Karpus S.G., Kuzmenko V.V., Shebeko K.V. Oxygen depletion of mylar under bombardment by 1.0 and $1.6 \mathrm{MeV}$ protons // East European Journal of Physics. - 2015. - Vol.2(1). - P.15-22. (in Russian)

5. Mitrofanov A.V., Apel P.Yu., Blonskaya I.V., Orelovich O.L. Dyfrakcionnye filtry na osnove poliimidnyh i polietilennaftalatnyh trekovyh membran [Diffraction filters on the basis of track polyimide and polyethylene teraphthalate membranes] //ZhTF. - 2006. - Vol.76. - Iss.9. - P.121-126. (in Russian)

6. Aleshin A.N., Gribanov A.V. Добродумов А.В., Суворов А.В., Шлимак И.С. Elektrofizicheskie svojstva plenok poliimida PM, podvergnutyh ionnoj bombardirovke [Electrophysical properties of PM polyimide films uder iom irradiation] // FTT. 1989. - Vol.31. - Iss.1. - P.12-18. (in Russian)

7. Sato S., Iwaki M. Target temperature dependence of sheet resistance and structure Ar-implanted diamonds // Nucl. Instr. and Meth. in Phys. Res. - 1988. - Vol.B32. - P.145-149.

8. Bondarenko V.N. Two-parameter model for irradiated polymer conductance vs ion beam fluency / The Journal of Kharkiv 
National University.-Physical series "Nuclei, Particles, Fields". - 2010. - No.899. - Issue 2/46/. - P.29-34. (in Russian)

9. Bondarenko V.N., Goncharov A.V., Kuzmenko V.V., Sukhostavets V.I. Study of polyimide electrical resistance vs ion beam fluence at irradiation by ions of nitrogen // The Journal of Kharkiv National University.-Physical series "Nuclei, Particles, Fields". - 2010. - No.933. - Issue 4/48/. - P.70-74. (in Russian)

10. Michelato P., Monaco L., Sertore D. In-vacuum temperature measurement of niobium components using infrared pyrometry during electron beam welding procedure // Proceedings of IPAC2013 (Shanghai, China). - P.2334-2336.

11. Boriskin V.N., Romanovsky S.K., Momot V.A., Titarenko Yu.A., Titov D.V., Uvarov V.L., Shevchenko V.A., Shelepko S.V. Optical monitoring the temperature of objects irradiated at an electron accelerator // Problems of atomic science and technology. Series "Nuclear Physics Investigations". - Kharkov: NSC KIPT. - 2015. - No.6(100). - P.105-107.

12. Bondarenko V.N., Glazunov L.S., Goncharov A.V., Zats A.V., Kolot V.Ya., Kuzmenko V.V., Levenets V.V., Omelnik A.P., Pystryak V.M., Sukhostavets V.I., Schur A.A. Analiticheskaya ustanovka dlya yaderno-fizicheskih metodov analiza [Analytical facility for ion-beam analysis] // Nauchnye vedomosti (Belgorodskiy gosudarstvennyy universitet), Series "Fizika". - 2001. No.2(15). - P.86-92. (in Russian)

13. 572X_gseng0000.pdf (http://www.fluke.com).

14. The Stopping and Range of Ions in Matter http:// www.srim.org

15. Vaysburd D.I., Pichugin V.F., Chebodayev M.I. Metodika opredeleniya termicheskogo soprotivleniya kontakta dielektrikpodlozhka pri intensivnyh rezhimah oblucheniya dielektrika [Techniques of thermal resistance of dielectric/substrate contact at intensive irradiation regime of dielectric] // Izv. vuzov. Fizika. - 2001. - No.12. - P.36-43. (in Russian)

16. Boyko V.I., Skvorcov V.A., Fortov V.Ye., Shamanin I.V. Vzaimodejstviye impulsnyh puchkov zaryazennyh chastic s veschestvom [Interaction of bunche charged particles beams with substance].-Moskow.: FIZMATLIT, 2003.-288p. (in Russian)

17. Lambert D.K. Thermal conductivity 21. Edited by C.J. Kremers and H.A. Fine. - N.Y.: Plenum Press, 1990. - P.209-219.

18. Quaranta A., Carturan S., Maggioni G., Milazzo P.M., Abbondano U., Della Mea G., Gramegna F., and Pieri U. Polyimidebased scntillation thin films // IEEE Trans. Nucl. Sci. - 2001. - Vol.48. - No.2. - P.219-224. 